\title{
Systematic Review of the Relationship between Acute and Late Gastrointestinal Toxicity after Radiotherapy for Prostate Cancer
}

\author{
Matthew Sean Peach, ${ }^{1}$ Timothy N. Showalter, ${ }^{1}$ and Nitin Ohri ${ }^{2}$ \\ ${ }^{1}$ Department of Radiation Oncology, University of Virginia School of Medicine, Charlottesville, VA 22908, USA \\ ${ }^{2}$ Department of Radiation Oncology, Montefiore Medical Center, Albert Einstein College of Medicine, Bronx, NY 10467, USA \\ Correspondence should be addressed to Nitin Ohri; ohri.nitin@gmail.com
}

Received 1 October 2015; Accepted 15 November 2015

Academic Editor: Robert Gardiner

Copyright (c) 2015 Matthew Sean Peach et al. This is an open access article distributed under the Creative Commons Attribution License, which permits unrestricted use, distribution, and reproduction in any medium, provided the original work is properly cited.

\begin{abstract}
A small but meaningful percentage of men who are treated with external beam radiation therapy for prostate cancer will develop late gastrointestinal toxicity. While numerous strategies to prevent gastrointestinal injury have been studied, clinical trials concentrating on late toxicity have been difficult to carry out. Identification of subjects at high risk for late gastrointestinal injury could allow toxicity prevention trials to be performed using reasonable sample sizes. Acute radiation therapy toxicity has been shown to predict late toxicity in several organ systems. Late toxicities may occur as a consequential effect of acute injury. In this systematic review of published reports, we found that late gastrointestinal toxicity following prostate radiotherapy seems to be statistically and potentially causally related to acute gastrointestinal morbidity as a consequential effect. We submit that acute gastrointestinal toxicity may be used to identify at-risk patients who may benefit from additional attention for medical interventions and close follow-up to prevent late toxicity. Acute gastrointestinal toxicity could also be explored as a surrogate endpoint for late effects in prospective trials.
\end{abstract}

\section{Introduction}

Prostate cancer is the most commonly diagnosed noncutaneous malignancy in men in developed countries. Definitive external beam radiotherapy (RT) is a treatment option for the majority of patients who present with localized disease. Additionally, RT may be offered after radical prostatectomy for patients whose pathology demonstrates adverse pathologic features or as salvage therapy for recurrent disease after surgery.

Regardless of the treatment technique used, RT for prostate cancer exposes a portion of the lower gastrointestinal (GI) tract to ionizing radiation and consequently carries a risk of GI toxicity. GI toxicity is categorized as occurring within two possible phases: acute (typically within 3 months of treatment) and late (more than 3 months after treatment) $[1,2]$. Symptoms can range from a mild increase in bowel movement frequency to more severe complications such as rectal bleeding, pain, or fistula. The acute phase of RT injury is characterized by inflammation in response to therapy, while the late phase is characterized by fibrosis and sclerosis within the GI tract [3]. While mild to moderate acute GI toxicity is more common than late toxicity, the potential permanent impact of late GI toxicity is thought to bear more clinical significance.

In an analysis of 35 studies including nearly 12,000 patients, rates of moderate (generally grade $\geq 2$ ) and severe (grade $\geq 3$ ) late GI toxicity following definitive external beam RT for prostate cancer were $15 \%$ and $2 \%$, respectively [4]. Dose-escalated RT, which has been shown to improve disease control rates [5] and is now standard of care, increases the risk of late GI toxicity [4-7]. Reported rates of late GI toxicity appear to be decreased when dose-escalated RT is delivered using advanced treatment techniques such as intensity-modulated radiotherapy (IMRT) [4]. Other measures to limit GI toxicity, such as the administration of radioprotective medications [8] or the use of spacers to separate the prostate and rectum [9], are being explored. Randomized clinical trials, 


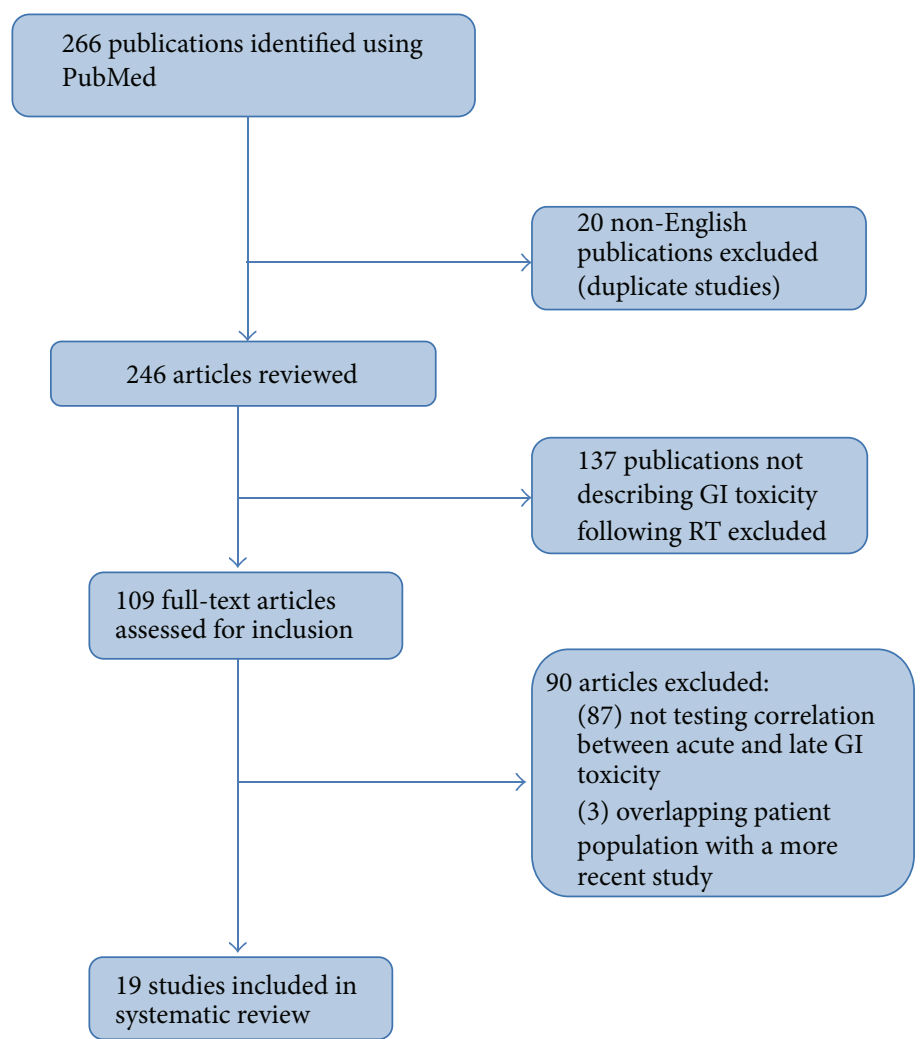

FIGURE 1: Selection strategy for systematic review of the published literature evaluating the relationship between acute and late gastrointestinal toxicity following prostate radiation therapy.

with large numbers of patients and lengthy follow-up, will be required to establish the efficacy of these toxicity prevention strategies.

There may be a consequential relationship between temporary acute GI toxicity and permanent late GI toxicity in prostate cancer patients treated with RT [10]. In this paper, we perform a systematic review to characterize the relationship between acute and late GI complications from prostate RT. We detail mechanisms by which acute toxicity may lead to consequential late effects. Finally, we explore the possibility of exploiting this connection for the identification of patients at risk of late GI toxicity and for the development of novel clinical trials for toxicity prevention.

\section{Methods}

2.1. Study Selection. We searched PubMed (http://www.ncbi .nlm.nih.gov/pubmed/) on May 1, 2013, for the terms "radiation therapy", "late", "early", and "side effects", with no limits placed on publication date. Duplicates and non-English language articles were excluded, and abstracts of all remaining manuscripts were read. Articles focusing on acute and late toxicity from external beam RT for prostate cancer were selected and examined in detail. Studies that examined the potential relationship between acute and late GI sequelae prostate RT were included in the final analysis. When two reports seemed to describe overlapping patient populations, the most recent publication was utilized for this analysis.
2.2. Data Extraction and Clinical Endpoints. Data abstraction was conducted according to the Preferred Reporting Items for Systematic Reviews and Meta-analyses (PRISMA) statement [11]. For each study, the following information was extracted: name of the first author, year of publication, name of the clinical trial (if applicable), sample size, and RT protocol. The primary measure of interest for this analysis was the association between late GI toxicity and acute GI toxicity. Hazard ratios, risk ratios, correlation coefficients, and other statistical measures describing the relationship between acute and late GI toxicity were extracted. Our preliminary analysis indicated that available data were not appropriate for metaanalysis, so we proceeded to perform a PRISMA-style systematic review.

\section{Results}

3.1. Study Selection. Search results are summarized in Figure 1. Our initial search yielded 266 results. Removal of duplicates and non-English language manuscripts reduced this number to 246.109 papers met initial eligibility criteria and were read at full length to determine if statistical tests for a link between acute and late GI toxicity were reported. Most of the papers were eliminated for merely reporting rates of acute and/or late GI toxicity in the study population. Others were excluded because they combined GI and genitourinary effects in their analyses. Three papers described patient populations that were likely included in subsequent reports 
from the same institutions. In total, 19 manuscripts met all eligibility requirements and were included in this report.

Ten manuscripts reported results from prospective trials [12-21] (Table 1), and nine were retrospective reviews of institutional experiences or databases [22-30] (Table 2). The trials that examined acute and late side effects involved a variety of techniques including conventional prostate RT [19], 3D conformal RT (3DCRT) [14, 15, 17, 24, 25, 30], high dose intensity modulated RT (IMRT) [27], hypofractionated IMRT [20, 23], combination brachytherapy + IMRT [29], and a mixed population treated with IMRT and 3DCRT [28]. Comparisons were also made in the manuscripts between different techniques including 3DCRT versus conventional RT [18]; 3DCRT dose escalation versus standard dose RT [12, 21]; 3DCRT versus hypofractionated 3DCRT [13]; a varied experience of 3DCRT and conventional RT \pm ADT [22]; high dose IMRT versus high dose 3DCRT [26]; and high dose IMRT \pm whole pelvis RT [16] (Table 1). The primary objective of most papers was to examine clinical factors leading to the development of acute GI and late GI toxicity [12-14, 16-18, 22, $28,30]$, while some specifically focused on the relationship between acute and late side effects $[14,19,21,30]$. The goal of other manuscripts was to simply report acute and late effects of a particular treatment $[15,20,21,23-27,29]$. Some of these studies tested medications to decrease toxicity, including rectal prostaglandin administration [15] and rectal sucralfate [19].

3.2. Evidence of Association between Acute and Late GI Toxicity. The manuscripts specifically looking for associations between acute and late effects demonstrated mild to strong correlations in various aspects of GI toxicity. Pinkawa et al. reported that acute bowel bothersome scores were associated with poor long-term bowel bothersome scores on univariate analysis, although with a HR of only 1.05; this relationship was not statistically significant in a multivariate analysis that accounted for RT dose and volume parameters [14]. Moderate to strong associations were found between multiple aspects of acute and late GI toxicity by Heemsbergen et al., where multivariable analysis suggested minimal contribution of RT dose and volume effects [21]. Acute proctitis was strongly (HR 2.9) associated with long-term diarrhea, defined as $\geq 6$ stools a day. Acute mucosal discharge was predictive of later use of incontinence pads (HR 2.1). Interestingly, the authors note that more objective factors of GI toxicity such as bleeding not included in RTOG had a stronger correlation. This suggests that a different scoring scheme may better demonstrate consequential GI toxicity relationships [21]. Perhaps the strongest evidence of this relationship came from O'Brien et al., who found that grade $\geq 2$ acute rectal pain was associated with grade $\geq 2$ late rectal toxicity with a HR of 3.4 [19]. However this manuscript did not examine dosimetric parameters, so observations were not adjusted for potential RT dose-volume interactions.

The strongest findings attesting to the relationship of acute and late GI toxicity came from manuscripts not primarily interested in determining this effect. Zelefsky et al., while specifically looking at late toxicity response to 3DCRT dose escalation in 1571 participants, found that acute grade $\geq 2$
GI toxicity was a strong predictor of late grade $\geq 2$ GI toxicity (HR 6.95, $p<0.001$ ). There was little contribution from dosimetric factors on multivariate analysis [28]. A similarly strong relationship was found between acute grade $\geq 2$ proctitis and late grade $\geq 1$ GI toxicity (OR 6.05, $p=0.03$ ) in a trial looking at the effect of misoprostol suppository on late rectal toxicity following 3DCRT [15]. Further, multivariate examination of late GI toxicity in over 100 patients subjected to high dose 3DCRT demonstrated that grade $\geq 2$ acute fecal incontinence was associated with chronic/late grade $\geq 2$ fecal incontinence (OR 4.43, $p=0.004$ ) and a stronger relationship for acute grade 3 fecal incontinence (OR 6.9, $p=0.001$ ) [17]. Of the remaining papers, most of the correlations were mild to moderate or were not significant once placed in multivariate analysis with dosimetric considerations (Tables 1 and 2).

3.3. Mechanism for Associations. With some exceptions [13, $18,19,21,30]$, most of the manuscripts that demonstrated an association between acute and late toxicities did not delve into the mechanism behind their findings. Koper et al. briefly discussed that their findings were most likely the result of consequential effects [18], namely, that acute toxicity leads to inflammation, leading to leakage of intestinal contents and eventual fibrosis manifesting as late toxicity. Alternatively, Arcangeli et al. concluded that the association between acute and late toxicity was evidence for a consequential mechanism but instead was a result of shared dosimetric risk factors. Interestingly, the association between acute and late effects observed in their conventionally fractionated RT arm was not observed in their hypofractionated RT arm [13].

Complementing their thorough data analysis, Heemsbergen et al. provided a more comprehensive explanation of the observed relationship between acute and late toxicity [21]. The authors put forth two possible mechanisms: the first a simple dose-volume relationship, and the second a continuum of consequential damage as has been observed in animal models [31]. In the former mechanism, severity of acute and late GI toxicity may correlate due to independent dose-volume effects on the RT at each time frame. Having the benefit of a large patient population, adequate followup, and uniform characterization of acute and late toxicity, the authors found on multivariate analysis that acute toxicity remained independently associated with late effects after adjusting for dosimetric variables and concluded that the relationship was most likely a combination of consequential effects and some dose volume effects. Of note, the group indicated that the acute RTOG score was not the most correlative factor; rather, tracking of individual GI symptoms was more revealing of the consequential pattern [21].

Heemsbergen indicated that other studies looking at acute-late correlation suffered from a lack of dose-volume considerations in their analysis [21]. This included the work of O'Brien et al. whose authors concede to not including dosimetric data but attest to dose-volume effects not contributing to acute toxicity in another study of a similar population and technique [19]. Koper et al. [18] offer alternative explanations to the observed correlation including inherent properties in individuals, such as yet described genetics or comorbidities that lead to greater tissue sensitivity to radiation both acutely 


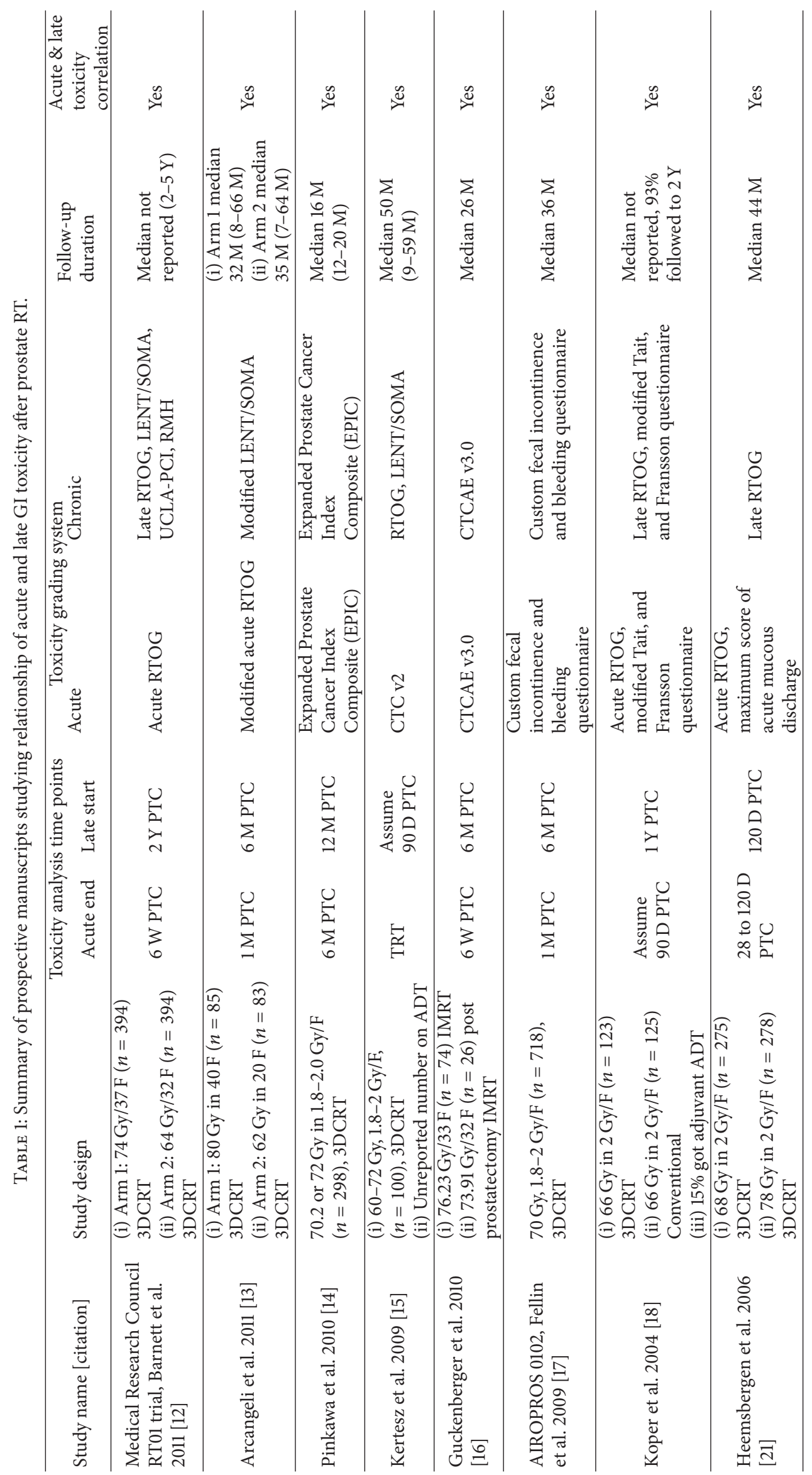




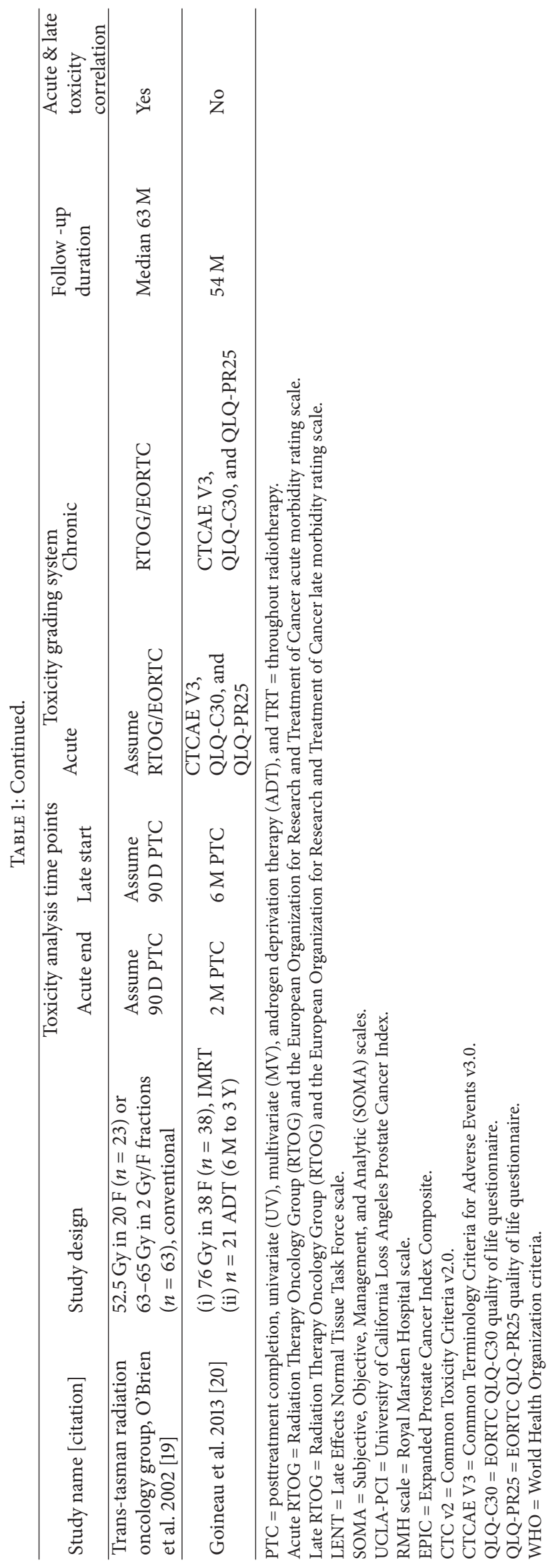




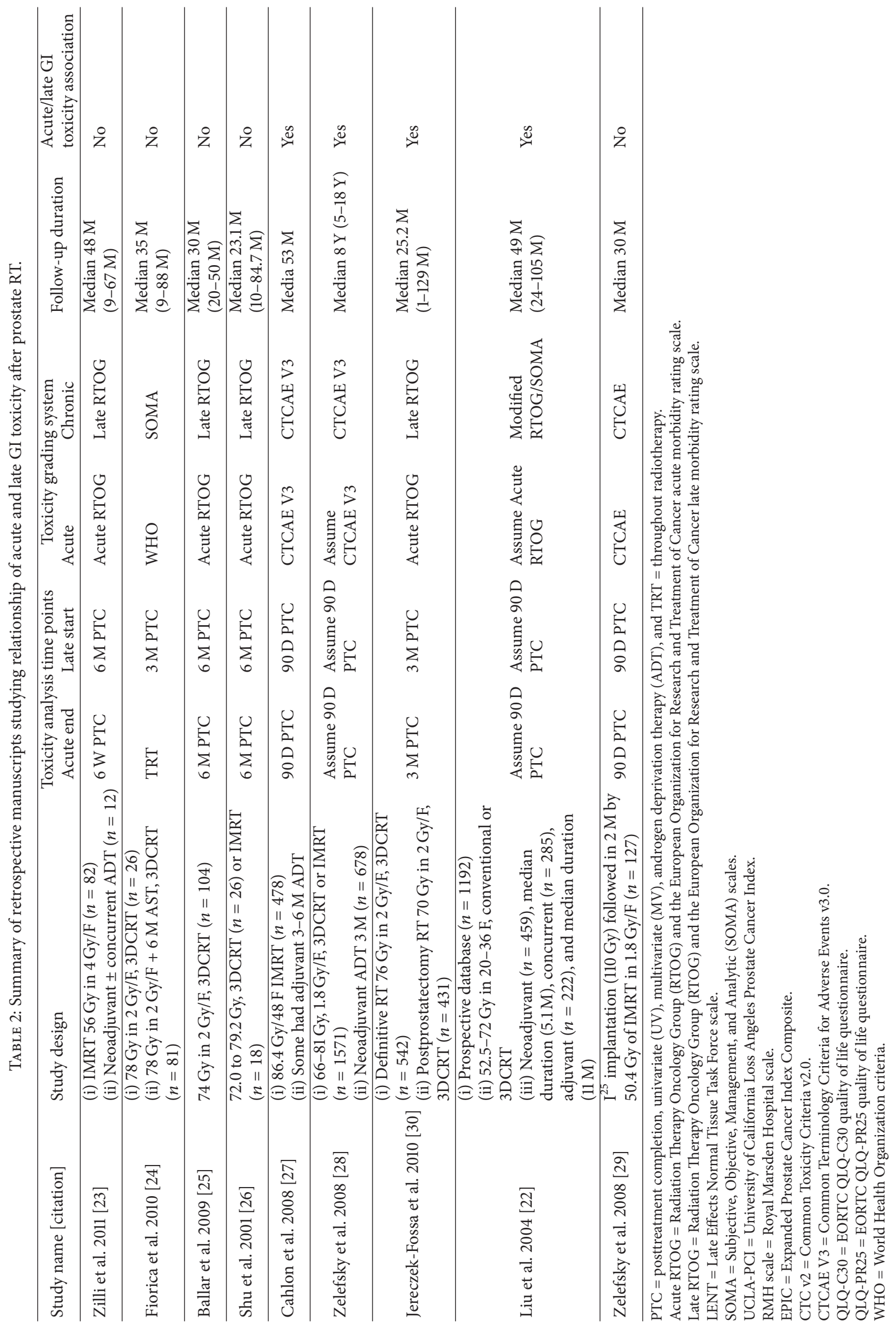


and chronically. A different explanation offered by Heemsbergen et al. is that some patients are more likely to communicate their symptoms and thus will verbalize both acute and late side effects alike [21]. However, the authors felt this theory was unlikely, as more objective findings such as acute and late rectal bleeding demonstrated strong associations [21]. The article by Jereczek-Fossa et al. was the most recent manuscript to thoroughly expand upon a proposed mechanism behind their demonstrated acute-late GI toxicity association [30]. The group believed, given the lack of prostate dose influence on late toxicity in a cohort of nearly 100 patients, that the association stemmed from consequential effects initiated by damage to the GI mucosa in the acute phase, citing works looking at consequential rectal toxicity in cervical cancer. Additionally, the authors asserted that age factored into the development of consequential damage when applied to multivariable analysis, in particular affecting acute toxicity as a result of comorbidities or an indirect effect of treatment decision-making [30].

Although not identified in the literature review, we are aware of the work by Campostrini et al. that showed more robust evidence of pathological consequential toxicity in humans [32]. Their group followed the progress of 130 patients from immediately after prostate RT and throughout the late period endoscopically (median follow-up of 84 months). It was noted that acute damage affected both the rectum and the anal canal macroscopically, with the most notable finding of hemorrhoid congestion, which was a major contributor to acute rectal bleeding. Interestingly, two patients had acute proctoscopic findings that were not manifested clinically. The finding of clinical and/or proctoscopic acute proctitis was strongly predictive of late toxicity (HR 5.6. 95\% CI 2.1-15.2, $p=0.001$ ) on multivariate analysis that incorporated dosimetric parameters [32].

3.4. Difficulties in Reporting the Correlation between Acute and Late Toxicities. Some of the authors of the manuscripts that did not observe a correlation between acute and late toxicities commented on the lack of findings, when viewed from the perspective of contradictory findings. Ballar et al. attributed the observed lack of correlation to a lower than normal acute toxicity found in their particular study compared to others [25]. Similarly, Zelefesky and colleagues manuscript did not identify a relationship between acute and late toxicities after combination brachytherapy and external beam RT, and they stated that this was likely due to fewer acute and late side effects than found in most similar studies [29]. Likewise, some manuscripts that did observe a relationship between acute and late toxicities also indicated potential study shortcomings that would underestimate the true consequential effect, such as short follow-up and newer RT techniques including IMRT that may cause less severe toxicity in both the acute and late setting [30]. Jereczek-Fossa et al. also noted that retrospective studies may suffer from underreported acute and late GI toxicity rates, complicated by the complexity of reporting late term side effects in prostate RT [30]. It has also been suggested that physician-based toxicity scoring, rather than patient-directed assessments, may introduce significant reporting bias [23].

\section{Discussion}

4.1. Acute Toxicity Is Predictive of Late Toxicity. The heterogeneity of the available data precludes quantitative synthesis in a formal meta-analysis, but we believe that the findings from this review shed significant light on the relationship between acute and late GI complications from prostate RT. Thirteen of the 19 studies that met inclusion criteria demonstrated an association between acute and late complications. Reports where no such associations were found tended to have a significantly smaller sample size than "positive" studies. Restricting our analysis to series with at least 200 subjects, for example, would leave nine remaining studies, all of which report a statistically significant link between acute and late complications. We therefore conclude that the overwhelming majority of the published evidence supports the presence of an association between acute and late GI toxicity following RT for prostate cancer.

Campostrini et al. provided strong evidence of pathologically confirmed acute toxicity as a significant predictor of late GI toxicity, even when dosimetric parameters as well as RT technique are taken into account. These findings are further bolstered by animal studies showing a stepwise pathologic progression from acute to late effects $[33,34]$. Therefore, the trends found in this systematic review of clinical studies, combined with observations from animal models, support a second important conclusion: acute toxicity may serve as an appropriate surrogate for late GI toxicity as a way to identify patients at high risk of developing permanent late GI toxicity, potentially for clinical trials of novel therapies intended to prevent development of consequential late GI toxicity, and as a surrogate endpoint for clinical trials.

4.2. Significance of Consequential Effect for Research. Since clinically significant late GI toxicity occurs in a minority of patients, clinical trials of medical interventions designed to prevent GI toxicity after prostate RT may require an excessively large sample size, if the trial designs are such that any prostate RT patient is eligible. However, establishing a consequential relationship between acute and late GI toxicity presents the opportunity to develop more efficient trial designs by focusing on a high-risk population. If acute GI toxicity is used as an eligibility criterion, which would restrict the study population to those at highest risk of late GI toxicity, a candidate medical intervention could be studied in clinical trial with a higher likelihood of identifying an effective strategy to reduce late GI toxicity. For example, if the study population has a $40 \%$ risk of late GI toxicity after IMRT (based on including only patients with significant acute toxicity), then a randomized, controlled trial of intervention versus placebo with a sample size of just 100 subjects would have $71 \%$ power to detect a $50 \%$ reduction in late toxicity events (personal communication, Nolan Wages, Ph.D.). If the baseline toxicity risk was $10 \%$ and all other parameters were unchanged, the study would only have a power of $25 \%$. Notably, this hypothetical example is potentially realistic, based on the available evidence identified in this report: assuming a $15 \%$ average risk of grade 2 or higher late GI toxicity [4] and a three- to sixfold increase in rates of late 
GI toxicity among patients with grade 2 or higher acute GI toxicity $[19,21,28]$, the risk of late toxicity among those patients with acute toxicity would be at least $40 \%$ and likely much higher.

The consequential nature of GI toxicity could be therefore exploited in research trials looking at interventions to avoid late toxicity in a number of ways:

(1) In future studies, acute toxicity may be used as a surrogate endpoint for late toxicity. This could decrease the duration and sample size required for prospective trials.

(2) Patients who demonstrate acute toxicity can be selected for long-term studies of late toxicity.

(3) Previously treated cohorts for which acute toxicity data are recorded can be assessed selectively for late toxicity, sampling only those patients who demonstrated acute GI toxicity.

4.3. Future Potential Studies: Pharmacological. With the above study framework, there are a number of pharmacological and nonpharmacological interventions than can be explored, some of which have already demonstrated the ability to prevent GI toxicity in pelvic radiation. This topic has been detailed in several recent reviews [35-37]. In regard to pharmacological interventions, the Cochrane review by Ali and Habib best summarizes the available options including such interventions as aminosalicylic acid (ASA) derivatives, sucralfate, arginine, vitamin E, probiotics, misoprostol, short chain fatty acid enemas, corticosteroids, cholestyramine, vitamin A, estrogen/progesterone, and octreotide [38]. Highlights from that review included manuscripts demonstrating the prevention of acute GI toxicity with oral sulphasalazine in prostate RT [39], as well as a decrease in acute and late GI toxicity when oral sucralfate is applied during and after RT for prostate and bladder cancer [40]. However, the previously cited work [19] and an earlier manuscript [41] by O'Brien et al. counter these findings when sucralfate is applied as a suppository during prostate RT in studies of similar size. Lastly, Ali and Habib referenced a small study showing that misoprostol suppositories applied before prostate external beam RT had protective properties [38]. Again, works encountered in this systematic review with larger numbers of participants did not find any benefit of misoprostol suppositories in the acute and late term [15].

There are other promising potential pharmacological compounds for preventing GI toxicity during pelvic RT. In regard to ASA derivatives, patients randomized to oral balsalazide during prostate RT achieved a CTC v2.0 prostate index of 35.3 versus 74.1 in placebo at two weeks after therapy $(p=0.04)$ [42]. However, a trial arm examining the rectal application of the ASA derivative mesalazine was prematurely terminated because of increased acute toxicity during prostrate 3DCRT in comparison to sucralfate enema control (HR 2.5, 95\% CI 1.1-5.7, and $p=0.03$ ) [43]. In the same study, no difference was found between sucralfate enema and hydrocortisone enema in preventing acute rectal toxicity [43]. Intrarectal application of the steroid beclomethasone in a more recent placebo controlled study did demonstrate an improved irritable bowel disease quality of life index, less rectal bleeding, and superior Vienna Rectoscopy Score up to 12 months following prostate RT [44]. Hyaluronic acid suppositories have also demonstrated the ability to decrease and delay acute radiation proctitis, according to RTOG scoring, when compared to historical prostate RT controls [45].

The free radical scavenger amifostine, when administered regularly during RT, has shown great potential in preventing acute rectal toxicity. In a randomized trial of 36 patients undergoing a mix of pelvic RT, intrarectal amifostine resulted in a significant decrease in RTOG score $(p<0.001)$, a decrease in LENT-SOMA score $(p=0.002)$, and improved proctoscopic tissue examination compared to controls [46]. More recent work has shown that increasing the dose of amifostine results in greater reduction of GI toxicity as determined by the EPIC bowel bothersome score during treatment and at 12 months after external prostate RT [47]. Lastly, the feasibility of rectal injections of Botox as a preventative measure against acute rectal toxicity has recently been studied based on Botox's effect on muscle spasticity, with future efficacy trials planned [48]. In total, there are a great deal more pharmaceutical compounds that have shown success in treating acute GI toxicity than treating late GI toxicity or preventing acute or late GI toxicity. Reassessment of some of these compounds as outlined above may be able to tease out greater usefulness in the above and other compounds.

4.4. Future Studies: Nonpharmacological. In regard to nonpharmacological strategies to prevent GI toxicity from pelvic $\mathrm{RT}$, rectal balloons have been studied for a number of years and are used routinely in some practices, particularly in proton beam RT. However, there appears to be only one small work to show decreased late GI toxicity, compared to treatment without a rectal balloon, as determined by proctoscopic assessment at two-year follow-up [49]. Rectal balloons have shown good patient compliance and tolerance [50]. The use of injectable spacers is an alternative approach that creates space between the prostate target volume and the rectum. For example, prospective evaluation with a polyethylene glycol hydrogel spacer in small study of 10 patients demonstrated very low acute GI toxicity. [51]. Collagen injections have also been used to increase prostate-rectal distance, resulting in a $50 \%$ decrease in the RT dose to the rectum [52]. Recently, a hybrid idea that in simulation appears to function well is the biodegradable interstitial balloon [53]. In comparison to some of the pharmacologically based preventative measures, studies of spacer interventions are for the most part lacking assessment on late term effects and would greatly benefit from study designs where acute toxicity was applied as a surrogate for late toxicity or used for patient selection in long-term trials.

\section{Conclusions}

Published data strongly support the presence of an association between acute and late GI toxicity following RT for prostate cancer. We suggest that acute GI toxicity may be used by physicians to identify patients who may benefit from 
personalized counseling and supportive care to address a high risk of permanent late GI toxicity. Furthermore, trials of strategies to prevent late morbidity might be enhanced by the preferential enrollment of subjects who develop acute toxicity in order to evaluate potential preventive strategies in a cohort of patients at high risk of late toxicity.

\section{Conflict of Interests}

The authors have no conflict of interests to report.

\section{References}

[1] A. Trotti, A. D. Colevas, A. Setser et al., "CTCAE v3.0: development of a comprehensive grading system for the adverse effects of cancer treatment," Seminars in Radiation Oncology, vol. 13, no. 3, pp. 176-181, 2003.

[2] J. D. Cox, J. Stetz, and T. F. Pajak, "Toxicity criteria of the radiation therapy oncology group (RTOG) and the european organization for research and treatment of cancer (RTOG)," International Journal of Radiation Oncology, Biology, Physics, vol. 31, no. 5, pp. 1341-1346, 1995.

[3] P. P. Tagkalidis and J. J. Tjandra, "Chronic radiation proctitis," ANZ Journal of Surgery, vol. 71, no. 4, pp. 230-237, 2001.

[4] N. Ohri, A. P. Dicker, and T. N. Showalter, "Late toxicity rates following definitive radiotherapy for prostate cancer," Canadian Journal of Urology, vol. 19, no. 4, pp. 6373-6380, 2012.

[5] G. A. Viani, E. J. Stefano, and S. L. Afonso, "Higher-than-conventional radiation doses in localized prostate cancer treatment: a meta-analysis of randomized, controlled trials," International Journal of Radiation Oncology Biology Physics, vol. 74, no. 5, pp. 1405-1418, 2009.

[6] J. M. Michalski, K. Bae, M. Roach et al., "Long-term toxicity following $3 \mathrm{D}$ conformal radiation therapy for prostate cancer from the rtog 9406 phase i/ii dose escalation study," International Journal of Radiation Oncology Biology Physics, vol. 76, no. 1, pp. 14-22, 2010.

[7] D. A. Kuban, S. L. Tucker, L. Dong et al., "Long-term results of the M. D. Anderson randomized dose-escalation trial for prostate cancer," International Journal of Radiation Oncology, Biology, Physics, vol. 70, no. 1, pp. 67-74, 2008.

[8] K. H. Katsanos, E. Briasoulis, P. Tsekeris et al., "Randomized phase II exploratory study of prophylactic amifostine in cancer patients who receive radical radiotherapy to the pelvis," Journal of Experimental and Clinical Cancer Research, vol. 29, article 68, 2010.

[9] M. Pinkawa, N. E. Corral, M. Caffaro et al., "Application of a spacer gel to optimize three-dimensional conformal and intensity modulated radiotherapy for prostate cancer," Radiotherapy and Oncology, vol. 100, no. 3, pp. 436-441, 2011.

[10] W. Dörr and J. H. Hendry, "Consequential late effects in normal tissues," Radiotherapy and Oncology, vol. 61, no. 3, pp. 223-231, 2001.

[11] D. Moher, A. Liberati, J. Tetzlaff, and D. G. Altman, "Preferred reporting items for systematic reviews and meta-analyses: the PRISMA statement," Annals of Internal Medicine, vol. 151, no. 4, pp. 264-269, W64, 2009.
[12] G. C. Barnett, G. De Meerleer, S. L. Gulliford, M. R. Sydes, R. M. Elliott, and D. P. Dearnaley, "The impact of clinical factors on the development of late radiation toxicity: results from the medical research council rt01 trial (isrctn47772397)," Clinical Oncology, vol. 23, no. 9, pp. 613-624, 2011.

[13] G. Arcangeli, J. Fowler, S. Gomellini et al., "Acute and late toxicity in a randomized trial of conventional versus hypofractionated three-dimensional conformal radiotherapy for prostate cancer," International Journal of Radiation Oncology Biology Physics, vol. 79, no. 4, pp. 1013-1021, 2011.

[14] M. Pinkawa, R. Holy, M. D. Piroth et al., "Consequential late effects after radiotherapy for prostate cancer-a prospective longitudinal quality of life study," Radiation Oncology, vol. 5, article 27, 2010.

[15] T. Kertesz, M. K. A. Herrmann, A. Zapf et al., "Effect of a prostaglandin - given rectally for prevention of radiation-induced acute proctitis-on late rectal toxicity. Results of a phase III randomized, placebo-controlled, double-blind study," Strahlentherapie und Onkologie, vol. 185, no. 9, pp. 596-602, 2009.

[16] M. Guckenberger, S. Ok, B. Polat, R. A. Sweeney, and M. Flentje, "Toxicity after intensity-modulated, image-guided radiotherapy for prostate cancer," Strahlentherapie und Onkologie, vol. 186, pp. 535-543, 2010.

[17] G. Fellin, C. Fiorino, T. Rancati et al., "Clinical and dosimetric predictors of late rectal toxicity after conformal radiation for localized prostate cancer: results of a large multicenter observational study," Radiotherapy and Oncology, vol. 93, no. 2, pp. 197-202, 2009.

[18] P. C. Koper, P. Jansen, W. van Putten et al., "Gastro-intestinal and genito-urinary morbidity after $3 \mathrm{D}$ conformal radiotherapy of prostate cancer: observations of a randomized trial," Radiotherapy and Oncology, vol. 73, no. 1, pp. 1-9, 2004.

[19] P. C. O’Brien, C. I. Franklin, M. G. Poulsen, D. J. Joseph, N. S. Spry, and J. W. Denham, "Acute symptoms, not rectally administered sucralfate, predict for late radiation proctitis: longer term follow-up of a phase III trial-Trans-Tasman Radiation Oncology Group," International Journal of Radiation Oncology Biology Physics, vol. 54, no. 2, pp. 442-449, 2002.

[20] A. Goineau, V. Marchand, J. Rigaud et al., "Prospective evaluation of quality of life 54 months after high-dose intensitymodulated radiotherapy for localized prostate cancer," Radiation Oncology, vol. 8, article 53, 2013.

[21] W. D. Heemsbergen, S. T. H. Peeters, P. C. M. Koper, M. S. Hoogeman, and J. V. Lebesque, "Acute and late gastrointestinal toxicity after radiotherapy in prostate cancer patients: consequential late damage," International Journal of Radiation Oncology Biology Physics, vol. 66, no. 1, pp. 3-10, 2006.

[22] M. Liu, T. Pickles, A. Agranovich et al., "Impact of neoadjuvant androgen ablation and other factors on late toxicity after external beam prostate radiotherapy," International Journal of Radiation Oncology Biology Physics, vol. 58, no. 1, pp. 59-67, 2004.

[23] T. Zilli, S. Jorcano, M. Rouzaud et al., "Twice-weekly hypofractionated intensity-modulated radiotherapy for localized prostate cancer with low-risk nodal involvement: toxicity and outcome from a dose escalation pilot study," International Journal of Radiation Oncology Biology Physics, vol. 81, no. 2, pp. 382-389, 2011.

[24] F. Fiorica, M. Berretta, C. Colosimo et al., "Safety and efficacy of radiotherapy treatment in elderly patients with localized prostate cancer: a retrospective analysis," Archives of Gerontology and Geriatrics, vol. 51, no. 3, pp. 277-282, 2010. 
[25] A. Ballar, M. D. Salvo, G. Lo, G. Ferrari, D. BeIdi, and M. Krengli, "Conformal radiotherapy of clinically localized prostate cancer: analysis of rectal and urinary toxicity and correlation with dose-volume parameters," Tumori, vol. 95, no. 2, pp. 160-168, 2009.

[26] H.-K. G. Shu, T. T. Lee, E. Vigneault et al., "Toxicity following high-dose three-dimensional conformal and intensity-modulated radiation therapy for clinically localized prostate cancer," Urology, vol. 57, no. 1, pp. 102-107, 2001.

[27] O. Cahlon, M. J. Zelefsky, A. Shippy et al., "Ultra-high dose (86.4 gy) imrt for localized prostate cancer: toxicity and biochemical outcomes," International Journal of Radiation Oncology Biology Physics, vol. 71, no. 2, pp. 330-337, 2008.

[28] M. J. Zelefsky, E. J. Levin, M. Hunt et al., "Incidence of late rectal and urinary toxicities after three-dimensional conformal radiotherapy and intensity-modulated radiotherapy for localized prostate cancer," International Journal of Radiation Oncology Biology Physics, vol. 70, no. 4, pp. 1124-1129, 2008.

[29] M. J. Zelefsky, M. A. Nedelka, Z.-L. Arican et al., "Combined brachytherapy with external beam radiotherapy for localized prostate cancer: reduced morbidity with an intraoperative brachytherapy planning technique and supplemental intensitymodulated radiation therapy," Brachytherapy, vol. 7, no. 1, pp. $1-6,2008$.

[30] B. A. Jereczek-Fossa, D. Zerini, C. Fodor et al., "Correlation between acute and late toxicity in 973 prostate cancer patients treated with three-dimensional conformal external beam radiotherapy," International Journal of Radiation Oncology Biology Physics, vol. 78, no. 1, pp. 26-34, 2010.

[31] J. W. Denham, M. Hauer-Jensen, T. Kron, and C. W. Langberg, "Treatment-time-dependence models of early and delayed radiation injury in rat small intestine," International Journal of Radiation Oncology Biology Physics, vol. 48, no. 3, pp. 871-887, 2000.

[32] F. Campostrini, R. Musola, G. Marchiaro, F. Lonardi, and G. Verlato, "Role of early proctoscopy in predicting late symptomatic proctitis after external radiation therapy for prostate carcinoma," International Journal of Radiation Oncology Biology Physics, vol. 85, no. 4, pp. 1031-1037, 2013.

[33] S. Kang, M. Chun, Y.-M. Jin et al., "A rat model for radiationinduced proctitis," Journal of Korean Medical Science, vol. 15, no. 6, pp. 682-689, 2000.

[34] Z. Symon, Y. Goldshmidt, O. Picard et al., "A murine model for the study of molecular pathogenesis of radiation proctitis," International Journal of Radiation Oncology Biology Physics, vol. 76, no. 1, pp. 242-250, 2010.

[35] B. Hanson, R. MacDonald, and A. Shaukat, "Endoscopic and medical therapy for chronic radiation proctopathy: a systematic review," Diseases of the Colon and Rectum, vol. 55, no. 10, pp. 1081-1095, 2012.

[36] A. K. Shadad, F. J. Sullivan, J. D. Martin, and L. J. Egan, "Gastrointestinal radiation injury: prevention and treatment," World Journal of Gastroenterology, vol. 19, no. 2, pp. 199-208, 2013.

[37] R. J. Gibson, D. M. K. Keefe, R. V. Lalla et al., "Systematic review of agents for the management of gastrointestinal mucositis in cancer patients," Supportive Care in Cancer, vol. 21, no. 1, pp. 313-326, 2013.

[38] S. Ali and I. Habib, "Pharmacological interventions for the prevention and treatment of radiation colitis, enteritis and proctitis," Cochrane Database of Systematic Reviews, 2011.
[39] D. Kiliç, I. Egehan, S. Özenirler, and A. Dursun, "Doubleblinded, randomized, placebo-controlled study to evaluate the effectiveness of sulphasalazine in preventing acute gastrointestinal complications due to radiotherapy," Radiotherapy and Oncology, vol. 57, no. 2, pp. 125-129, 2000.

[40] R. Henriksson, L. Franzen, and B. Littbrand, "Effects of sucralfate on acute and late bowel discomfort following radiotherapy of pelvic cancer," Journal of Clinical Oncology, vol. 10, no. 6, pp. 969-975, 1992.

[41] P. C. O’Brien, C. I. Franklin, K. B. G. Dear et al., "A phase III double-blind randomised study of rectal sucralfate suspension in the prevention of acute radiation proctitis," Radiotherapy and Oncology, vol. 45, no. 2, pp. 117-123, 1997.

[42] C. D. Jahraus, D. Bettenhausen, U. Malik, M. Sellitti, and W. H. St. Clair, "Prevention of acute radiation-induced proctosigmoiditis by balsalazide: a randomized, double-blind, placebo controlled trial in prostate cancer patients," International Journal of Radiation Oncology Biology Physics, vol. 63, no. 5, pp. 1483-1487, 2005.

[43] G. Sanguineti, P. Franzone, M. Marcenaro, F. Foppiano, and V. Vitale, "Sucralfate versus mesalazine versus hydrocortisone in the prevention of acute radiation proctitis during conformal radiotherapy for prostate carcinoma. A randomized study," Strahlentherapie und Onkologie, vol. 179, no. 7, pp. 464-470, 2003.

[44] L. Fuccio, A. Guido, L. Laterza et al., "Randomised clinical trial: preventive treatment with topical rectal beclomethasone dipropionate reduces post-radiation risk of bleeding in patients irradiated for prostate cancer," Alimentary Pharmacology and Therapeutics, vol. 34, no. 6, pp. 628-637, 2011.

[45] A. Stefanelli, G. Pascale, E. Rainieri et al., "Can we decrease the acute proctitis in prostate cancer patients using hyaluronic acid during radiation therapy: a prospective historically controlled clinical study," European Review for Medical and Pharmacological Sciences, vol. 16, no. 5, pp. 639-645, 2012.

[46] J. R. Kouvaris, V. Kouloulias, E. Malas et al., "Amifostine as radioprotective agent for the rectal mucosa during irradiation of pelvic tumors. A phase II randomized study using various toxicity scales and rectosigmoidoscopy," Strahlentherapie und Onkologie, vol. 179, no. 3, pp. 167-174, 2003.

[47] N. L. Simone, C. Ménard, B. P. Soule et al., "Intrarectal amifostine during external beam radiation therapy for prostate cancer produces significant improvements in quality of life measured by epic score," International Journal of Radiation Oncology Biology Physics, vol. 70, no. 1, pp. 90-95, 2008.

[48] T. Vuong, K. Waschke, T. Niazi et al., "The value of botox-a in acute radiation proctitis: results from a phase I/II study using a three-dimensional scoring system," International Journal of Radiation Oncology Biology Physics, vol. 80, no. 5, pp. 1505-1511, 2011.

[49] E. N. J. T. van Lin, J. Kristinsson, M. E. P. Philippens et al., "Reduced late rectal mucosal changes after prostate threedimensional conformal radiotherapy with endorectal balloon as observed in repeated endoscopy," International Journal of Radiation Oncology Biology Physics, vol. 67, no. 3, pp. 799-811, 2007.

[50] R. J. Smeenk, B. S. Teh, E. B. Butler, E. N. J. T. van Lin, and J. H. A. M. Kaanders, "Is there a role for endorectal balloons in prostate radiotherapy? A systematic review," Radiotherapy and Oncology, vol. 95, no. 3, pp. 277-282, 2010. 
[51] F. Eckert, S. Alloussi, F. Paulsen et al., "Prospective evaluation of a hydrogel spacer for rectal separation in dose-escalated intensity-modulated radiotherapy for clinically localized prostate cancer," BMC Cancer, vol. 13, article 27, 2013.

[52] W. R. Noyes, C. C. Hosford, and S. E. Schultz, "Human collagen injections to reduce rectal dose during radiotherapy," International Journal of Radiation Oncology, Biology, Physics, vol. 82, no. 5, pp. 1918-1922, 2012.

[53] C. Melchert, E. Gez, G. Bohlen et al., "Interstitial biodegradable balloon for reduced rectal dose during prostate radiotherapy: results of a virtual planning investigation based on the preand post-implant imaging data of an international multicenter study," Radiotherapy and Oncology, vol. 106, no. 2, pp. 210-214, 2013. 


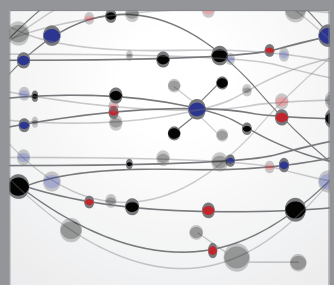

The Scientific World Journal
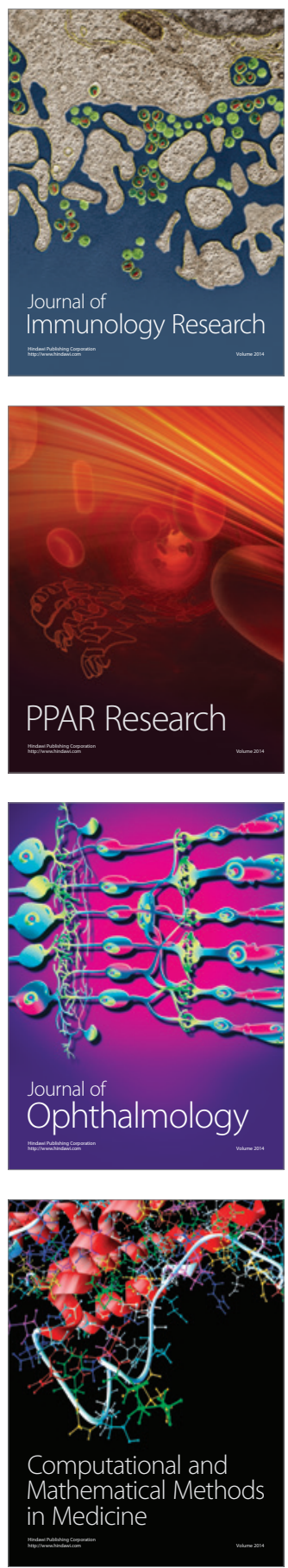

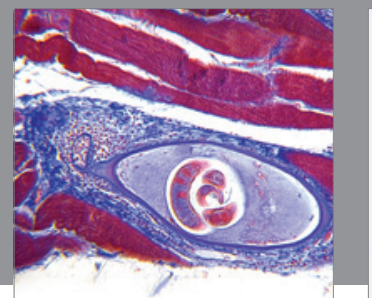

Gastroenterology

Research and Practice
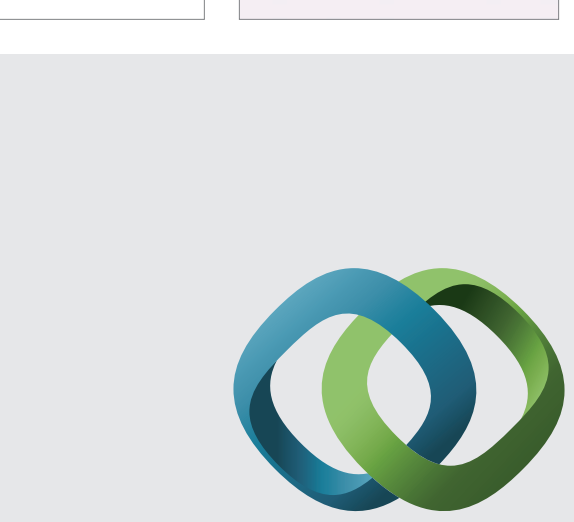

\section{Hindawi}

Submit your manuscripts at

http://www.hindawi.com
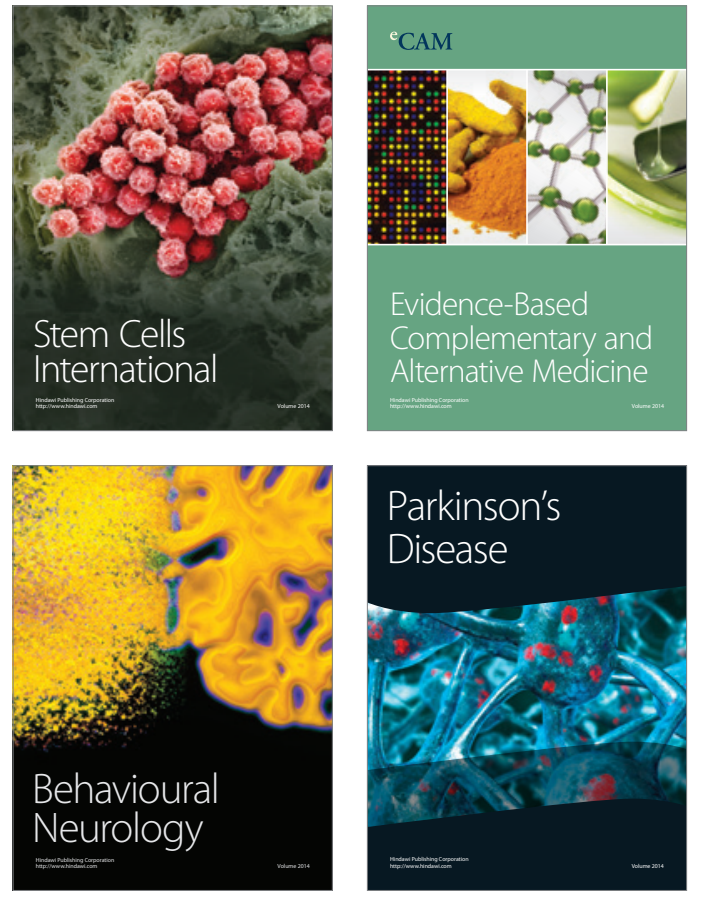
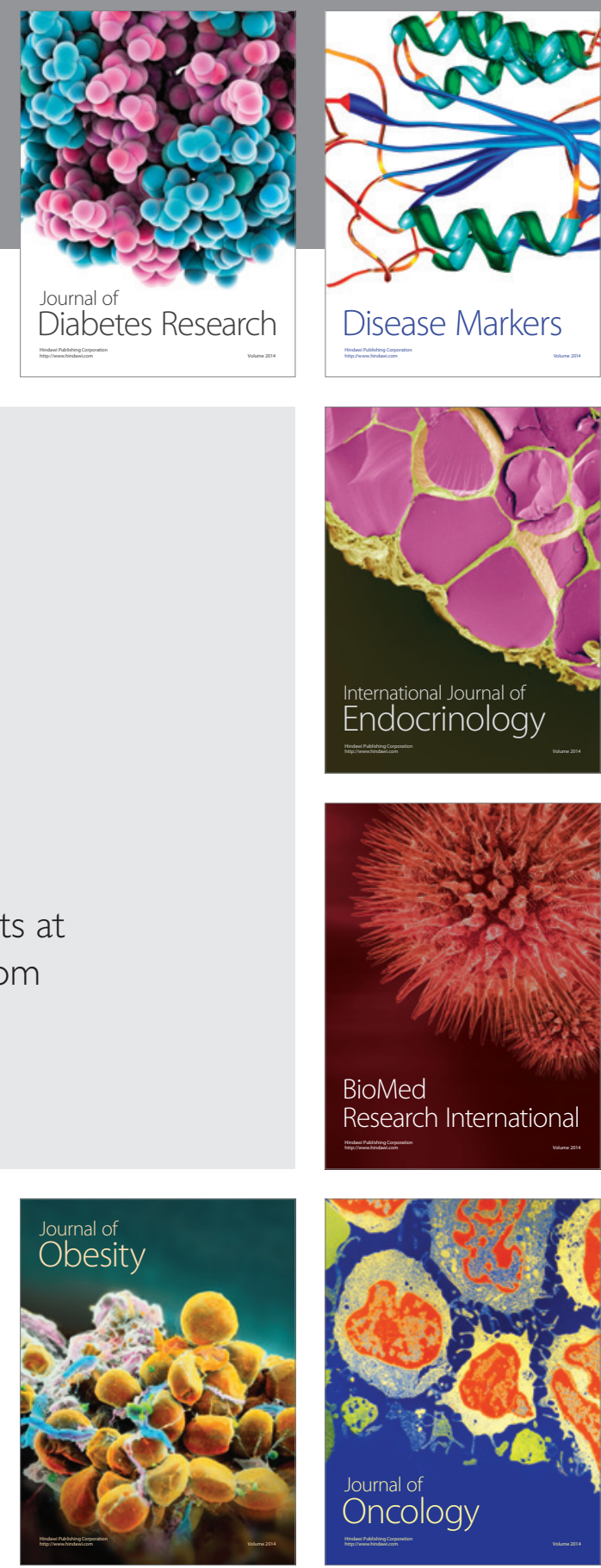

Disease Markers
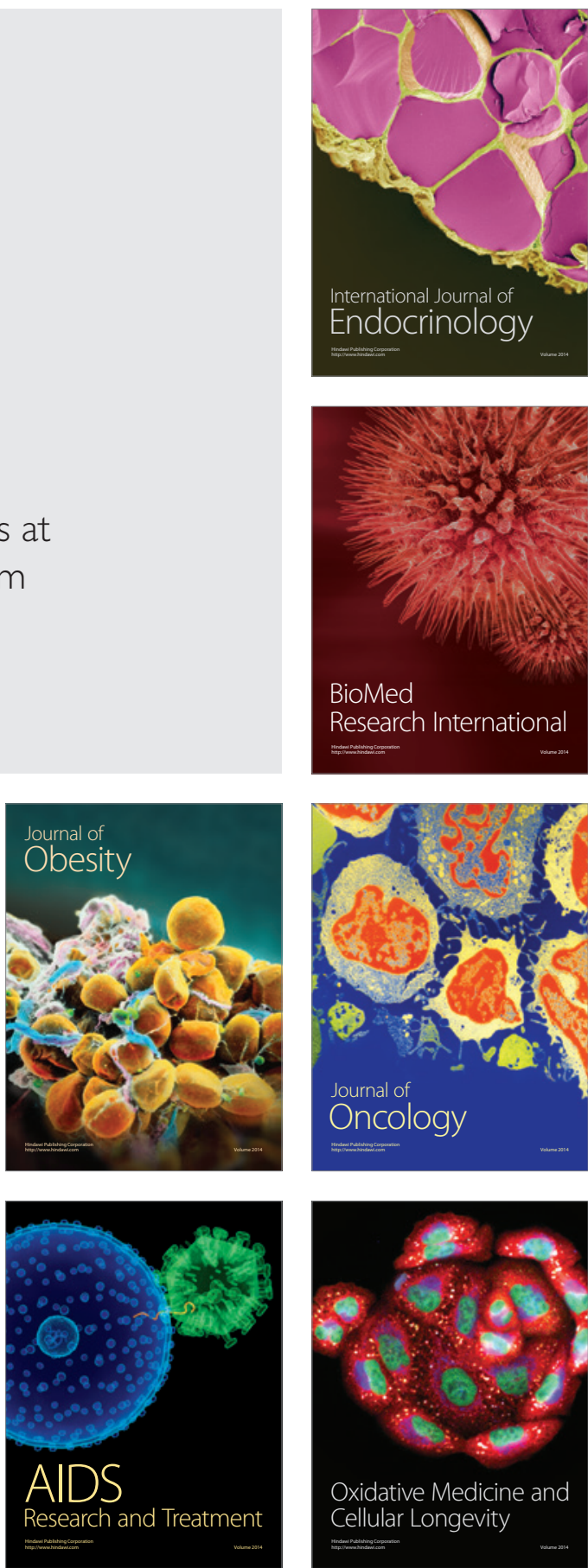